\title{
Profundizando en la educación científica: aspectos epistemológicos y metodológicos a tener en cuenta en la enseñanza
}

\section{Aprofundando a educação científica: aspectos epistemológicos e metodológicos a se considerar no ensino}

\section{Discussing science education: epistemological and methodological aspects to consider in teaching}

\author{
Carles Furió-Más ${ }^{1}$ \\ Cristina Furió-Gómez ${ }^{2}$ \\ Jordi Solbes-Matarredona ${ }^{3}$
}

\begin{abstract}
RESUMEN
El principal objetivo de este trabajo consiste en aproximar al profesorado hacia algunos logros de la investigación sobre la didáctica de las ciencias. En primer lugar, se muestra cómo la historia y epistemología de las Ciencias y, en particular, de la Química, está contribuyendo a la mejora de su enseñanza teniendo presentes algunos aspectos conceptuales y epistemológicos que pueden facilitar la alfabetización científica. En particular, se presentan ejemplos de construcción de conceptos clave en la Química escolar como el peso de los gases, la idea de sustancia y la de elemento químico. También se advierte al profesorado sobre algunos tópicos criticables sobre la
\end{abstract}

${ }^{1}$ Doctor en Ciencias Químicas. Departamento de Didáctica de las Ciencias Experimentales y Sociales de la Universidad de Valencia, España. E-mail: carles.furio@uv.es

${ }^{2}$ Doctora en Didáctica de las Ciencias Experimentales. Departamento de Didáctica de las Ciencias Experimentales y Sociales de la Universidad de Valencia, España. E-mail: cristina.furio@uv.es

${ }^{3}$ Doctor en Ciencias Físicas. Departamento de Didáctica de las Ciencias Experimentales y Sociales de la Universidad de Valencia, España. E-mail: jordi.solbes@uv.es 
naturaleza de la ciencia. En segundo lugar, se hace una breve presentación de las tradiciones más importantes de investigación sobre alfabetización científica y tecnológica: la del cambio conceptual, la sociocultural y la crítica. Finalmente se abordan las orientaciones metodológicas del modelo de aprendizaje como investigación orientada que ya se han utilizado con resultados satisfactorios en la enseñanza de temáticas específicas de las disciplinas de Física y Química.

Palabras-clave: didáctica de las ciencias; historia y epistemología de las ciencias; alfabetización científica y tecnológica; aprendizaje como investigación orientada; enseñanza de Física y Química.

\title{
RESUMO
}

O principal objetivo deste trabalho consiste em aproximar os professores de alguns resultados da investigação sobre a didática das ciências. Em primeiro lugar se mostra como a história e a epistemologia das ciências e, em particular, da Química, está contribuindo à melhoria de seu ensino, considerando alguns aspectos conceituais e epistemológicos que podem facilitar a alfabetização científica. Em particular, são apresentados exemplos da construção de conceitos chave na Química escolar como o peso dos gases, a noção de substância e a de elemento químico. Também se alerta os professores sobre alguns tópicos críticos sobre a natureza da ciência. Em segundo lugar, se faz uma breve apresentação das mais importantes tradições de investigação sobre alfabetização científica e tecnológica: a de mudança conceitual, a sociocultural e a crítica. Finalmente se abordam as orientações metodológicas do modelo de aprendizagem como investigação orientada que têm sido utilizadas com resultados satisfatórios no ensino de temáticas específicas das disciplinas Física e Química.

Palavras-chave: didática das ciências; história e epistemologia das ciências; alfabetização científica e tecnológica; aprendizagem como investigação orientada; ensino de Física e Química.

\begin{abstract}
The main objective of this paper is to present teachers with some of the achievements of research on science didactics. First, it shows how the history and epistemology of science, in particular in Chemistry, are contributing to improvements in teaching, considering the inclusion of conceptual and epistemological aspects that facilitate scientific literacy. In particular, examples in building key concepts in school Chemistry are presented, such as the weight of gases, the idea of substance and
\end{abstract}


chemical element. It also warns teachers of some of the critical topics about the nature of science. Secondly, a brief presentation of the major traditions of research on scientific and technological literacy is presented: the conceptual, the sociocultural and critical changes. Finally, some methodological guidelines of learning that have already been successfully used in teaching specific subjects in Physics and Chemistry, such as the oriented research model, are presented.

Keywords: science education; history and epistemology of science; scientific and technological literacy; learning as oriented research; Physics and Chemistry teaching.

\section{Introducción}

Desde la segunda mitad del siglo pasado se ha venido construyendo un campo propio de investigación de Didáctica de las Ciencias que trata de integrar conocimientos de dominios de investigación tan diferentes como los de la psicología del aprendizaje, la historia y epistemología de las ciencias, la pedagogía del proceso de enseñanza, la sociología, la antropología y la lingüística (ABELL; LEDERMAN, 2007). La meta fundamental de este nuevo dominio de investigación ha sido y sigue siendo mejorar el proceso de aprender ciencias, tratando de ayudar a los estudiantes a superar las dificultades que se les presentan mediante la enseñanza.

Esta convergencia de intereses en los estudios sobre el aprendizaje de las ciencias ha hecho que en los últimos 40 años esta investigación haya avanzado bastante en el sentido de que actualmente se acepte mayoritariamente que lograr una buena educación científica ha de consistir en desarrollar una alfabetización científica y tecnológica en el alumnado. Esta alfabetización es un término que utilizamos, en general, para designar las competencias científicas y tecnológicas (conocimientos, prácticas y valores) que esperamos que los alumnos desarrollen cuando aprenden ciencia. Desarrollo que se ha de iniciar en la escolarización obligatoria pero que ha de continuar a lo largo de toda la vida y que ha de formar parte importante de la cultura de cualquier ciudadano o ciudadana del siglo XXI con el fin de que pueda tomar decisiones fundamentadas sobre los cambios del mundo natural y, en particular, sobre los producidos por la actividad humana (LAUGKSCH, 2000). Es más, esta alfabetización científica y tecnológica ha sido declarada como factor esencial para el desarrollo de las personas y de los pueblos por instituciones internacionales como, por ejemplo, el National 
Research Council (1996). Y aunque desde hace más de 10 años disponemos de definiciones razonables de alfabetización científica y de cómo lograrla (BYBEE, 1997; NATIONAL RESEARCH COUNCIL, 2000), es muy frustrante comprobar mediante la investigación que la institución dedicada a la educación formal no ayuda a que la mayoría de los estudiantes aprendan ciencia con comprensión. La pregunta a plantearse es, pues: ¿Por qué no aprenden los estudiantes lo que los profesores tratamos de enseñarles? Un documento reciente que responde directamente a esta pregunta es el Informe de Rocard et al. (2008), Science Education Now: A renewed Pedagogy for the future of Europe. En él se alerta sobre el "peligro capital para el futuro de Europa" que supone la disminución de jóvenes que estudian ciencias. Este abandono escolar obstaculiza uno de los principales objetivos de la U.E: lograr en un futuro próximo una economía del conocimiento. En dicho informe se señala que: "Los orígenes de esta situación puede encontrarse en la manera como se enseña". Estamos de acuerdo con la importancia dada a los métodos de enseñanza aunque la investigación ya ha mostrado que el desinterés y abandono del alumnado de los estudios científicos es un fenómeno complejo, multicausal, en el que influyen, además de la enseñanza, otros factores como, por ejemplo, la imagen pública de la ciencia, las relaciones género-ciencia o la poca importancia dada por la política educativa a las ciencias en el curriculum escolar (SOLBES et al., 2007). En este trabajo trataremos de aproximar la investigación al profesorado con el fin de que adquieran conocimientos sobre las principales tradiciones de la investigación en didáctica de las ciencias que se han ido desarrollando sobre la alfabetización científica y tecnológica durante los últimos años. Y, en particular, como ha ido cambiando nuestra visión sobre como aprenden ciencias los alumnos, sobre la naturaleza de la ciencia (la alfabetización científica también depende de las ideas que tenemos los profesores sobre la ciencia) y sobre los métodos de enseñanza empleados para mejorar el aprendizaje. Hemos acotado este trabajo a los aspectos epistemológicos y metodológicos del proceso de enseñanza-aprendizaje siendo conscientes de que también son importantes otras dimensiones del proceso como son los aspectos axiológicos y actitudinales.

Por todo ello en este trabajo centraremos nuestra atención en mostrar, en primer lugar, como la historia y epistemología de las Ciencias y, en particular, de la Química, está contribuyendo a la mejora de su enseñanza teniendo presentes algunos aspectos conceptuales y epistemológicos que pueden facilitar el aprendizaje (MILLAR; OSBORNE, 1998; FURIÓ-MÁS, 2009). En segundo lugar, se hará una breve presentación de las tradiciones más importantes de investigación sobre alfabetización científica y tecnológica: la del cambio conceptual, la sociocultural y la crítica). Finalmente concretaremos las orientaciones metodológicas del modelo de aprendizaje como investigación orientada 
que hemos utilizado con resultados satisfactorios en la enseñanza de temáticas específicas de las disciplinas de Física y Química (GUISASOLA et al., 2008; OSUNA et al, 2007; FURIÓ et al., 2006).

\section{La historia y epistemología de la ciencia y las dificultades en el aprendizaje de la Química}

Es bien conocido el interés que tiene para el profesor saber cómo piensa el estudiante sobre cualquier contenido para tratar de enseñarlo en consecuencia, como indica la psicología cognitiva. Por otra parte, conviene recordar que a principios de la década de los 80 comenzó a construirse la tradición didáctica del aprendizaje como cambio conceptual basada inicialmente en el proceso piagetiano de asimilación y acomodación y en la aplicación de la historia y epistemología de la ciencia (HEC) como estrategia para mejorar la enseñanza y el aprendizaje científicos (FILLON, 1991). Como bien indican Erduran y Duschl (2004): "Esta relación entre cambio de teorías en la ciencia y desarrollo conceptual en el alumnado se popularizó gracias a la idea de Kuhn (1971) de que 'la ontogenia cognitiva recapitula la filogenia cientifica'. En efecto, la publicación del artículo de Posner et al. (1982) desempeñaron un papel clave en la formulación de la teoría del cambio conceptual”. En este sentido y aún admitiendo que este isomorfismo tiene serias limitaciones, presentaremos resultados de algunas investigaciones realizadas sobre semejanzas entre problemas habidos en la introducción de conceptos clave en la historia de la Química y dificultades actuales en el aprendizaje de la Química.

Partimos, pues, del supuesto de que conocer la HEC puede proporcionar al profesor ideas sobre cuáles pueden ser las principales dificultades que se pueden presentar en el aprendizaje previamente a la enseñanza. Recíprocamente, se pueden investigar empíricamente las dificultades de los estudiantes y bucear en los modelos históricos habidos en el desarrollo de la ciencia para ver si hay posibles isomorfismos al comparar los obstáculos epistemológicos encontrados en el aula y los problemas con que se encontraron los científicos en una época determinada de la historia. Presentaremos dos casos de paralelismos entre las dificultades de los estudiantes y los problemas que se tuvieron que superar por los científicos para llegar a configurar el modelo atómico de la materia: la aceptación de la materialidad de los gases ya que los filósofos griegos los consideraban como "materia rara" distinta a la "materia corpórea" y la introducción del concepto macroscópico de sustancia (pura) superando la idea aristotélica de que todos los materiales terrestres eran mezclas. 


\section{¿Pesan o no pesan los gases que nos rodean?}

En un trabajo anterior (FURIÓ et al., 1987) se investigó cuáles eran las ideas de los estudiantes sobre la materialidad de los gases. Es decir, si los gases se concebían con las mismas propiedades generales (masa, volumen, dureza, elasticidad, dilatación y contracción térmica, etc.) que la materia condensada, sólidos y líquidos. Consideramos en aquella investigación que una de las propiedades que mejor refleja la materialidad de un cuerpo es su masa, magnitud que habitualmente se confunde con el peso. Se presuponía que el alumnado, incluido el que había estudiado varios cursos de Química en la Secundaria y el Bachillerato, pensaría que los gases no pesan porque flotan y se van hacia arriba y, por tanto, el alumnado no los tendría en cuenta en fenómenos físicos como la vaporización, o químicos como la oxidación de hierro. Se utilizó un diseño experimental convergente a base de cuestionarios y entrevistas que se pasó a una muestra de 1200 estudiantes del antiguo Bachillerato y de C.O.U españoles (de 13 a 18 años). Los resultados obtenidos contrastaron la hipótesis de que los gases no pesan en, aproximadamente, las dos terceras partes de la muestra encuestada (70\%) (FURIÓ-MÁS; FURIÓ-GÓMEZ, 2009).

Se supuso que esta concepción espontánea de que los gases eran inmateriales podría tener antecedentes en alguna época histórica. Se realizó un análisis histórico y epistemológico de los principales problemas que tuvo que superar la Química de los siglos XVI al XVIII para llegar a poder construir el modelo atómico de la materia y llegamos a la conclusión de que dos de los principales logros fueron los siguientes:

* Aceptar que los gases macroscópicamente eran tan corpóreos como los sólidos y líquidos poniendo en cuestión el modelo aristotélico-escolástico hegemónico durante más de 20 siglos en el que la materia corpórea se diferenciaba de la materia rara (que contenía una forma casi espiritual que los griegos denominaron pneuma) (WANDERSEE; MINTZES; NOVAK, 1994).

* Cuestionar que todos los materiales del mundo terrestre eran impuros, esto es formados por mezclas de 4 elementos y 3 principios considerados como formas o cualidades. Ello condujo a introducir el concepto macroscópico de sustancia - en particular, el de compuesto químico - como material puro, no mezclado (con propiedades características) y, por tanto, opuesto al de mezcla (FURIÓ et al., 2000).

Así pues, en el primer caso la investigación ha puesto de manifiesto que existe cierta semejanza entre las concepciones del alumnado sobre la materialidad de los gases y los problemas históricos sobre el aire que hubo en la transición del modelo aristotélico-escolástico al modelo mecanicista de los siglos XVI al 
XVIII, inclusive. Recordamos brevemente a estos efectos que es en esta época cuando Torricelli, Pascal, y otros propusieron la hipótesis de que el aire pesaba porque vivíamos en el fondo de "un mar de aire" en contra de la hipótesis asumida por la mayoría de los filósofos de que "la naturaleza tenía horror al vacio". Con esta última hipótesis se explicaba, por ejemplo, que el agua de una botella invertida dentro de otro recipiente con agua no cayera. Este peso del aire de los filósofos mecánicos es la razón de que hoy sigamos hablando de la enorme presión atmosférica que gravita sobre nuestras cabezas y que pasa desapercibida incluso para los estudiantes de ciencias.

Otra investigación que nos planteamos fue estudiar si también había cierta semejanza entre las dificultades del alumnado en la conceptualización empírica de sustancia química y la construcción histórica de sustancia (pura) y compuesto químico durante los siglos XVII y XVIII.

¿Se presentan dificultades en la comprensión actual del concepto de sustancia química? ¿Cómo se construyó históricamente la conceptualización de sustancia $y$, en particular, la de compuesto químico?

En otras investigaciones (FURIÓ; BULLEJOS; DE MANUEL, 1994; DOMÍNGUEZ; FURIÓ-MÁS, 2001; FURIÓ; DOMÍNGUEZ, 2007) se han estudiado las dificultades del alumnado sobre la conceptualización de sustancia química (y, en particular, si confundían la idea de mezcla con la de sustancia compuesta). Se utilizaron cuestionarios y entrevistas en muestras significativas de estudiantes de Secundaria y Bachillerato (de 15 a 18 años) y se llegó a la conclusión de que la imagen química actual que tienen los estudiantes del mundo posee las siguientes características:

- La materia está formada por mezclas (o compuestos) de sustancias más simples (o elementos químicos).

- Identifican sustancias fijándose en sus propiedades cualitativas poco específicas (color, olor, sabor, etc.) que para ellos son las que suelen conservarse en los procesos químicos.

- No disponen de criterios adecuados para saber si un compuesto es una mezcla de sustancias simples o una sustancia única.

- No pueden diferenciar los cambios físicos de los químicos porque no disponen de criterios cuando no se conservan las propiedades cualitativas de las sustancias. En estos casos, la mayoría acepta de forma natural la transmutación de sustancias.

En los resultados encontrados en esta investigación cerca del $80 \%$ de los estudiantes de Química del último curso de Bachillerato (18 años) no logró res- 
ponder correctamente a preguntas donde tenían que decir cuáles materiales de entre varios (por ejemplo, aire, agua, granito, ondas de radio, etc.) consideraban que estaban formados por una única sustancia.

En este caso, el análisis histórico y epistemológico de cómo se llegó a construir científicamente el concepto de sustancia química fue más complejo que el de los gases. Se analizó el significado dado a la idea de sustancia en cada uno de los tres modelos históricos: el aristotélico-escolástico, el mecanicista ya indicado y el atomista del siglo XIX (JENSEN, 1998; KLEIN; LÉFEVRE, 2007). En el cuadro 1 se resumen las visiones históricas sobre cómo se suponía que estaban formados los materiales ordinarios (terrestres) y en el cuadro 2 cómo se conceptualizó la idea de sustancia en cada uno de aquellos modelos.

\begin{tabular}{|l|l|l|}
\hline \multicolumn{1}{|c|}{ Modelo escolástico } & \multicolumn{1}{|c|}{ Modelo mecanicista } & \multicolumn{1}{c|}{ Modelo atomista } \\
\hline Loa cuerpos terrestres están & La materia se presenta en & Los cuerpos se clasifican \\
formados por mezclas de 4 & forma de: mezclas, "cuer- & en: mezclas y sustancias. \\
elementos y 3 principios & pos perfectamente mezcla- & Las sustancias pueden ser \\
abstractos (no reales). & $\begin{array}{l}\text { dos" y “cuerpos perfecta- } \\
\text { mente sin mezcla". }\end{array}$ & simples o compuestas. \\
\hline
\end{tabular}

Cuadro 1 - Tres visiones históricas de cómo está formada la materia ordinaria.

\begin{tabular}{|l|l|l|}
\hline \multicolumn{1}{|c|}{ Modelo escolástico } & \multicolumn{1}{|c|}{ Modelo mecanicista } & \multicolumn{1}{|c|}{ Modelo atomista } \\
\hline No existe la idea de sustancia & La sustancia se define & Se asume la idea empirista \\
pura dado que la materia & empíricamente como cuerpo & de sustancia y se interpreta \\
"real" es impura. O sea, está & puro, no mezclado, que tiene & microscópicamente como \\
formada por una mezcla de los & un conjunto de propiedades & formada por conjunto de \\
4 elementos (aire, agua, tierra & características fijas. Se opone & particulas iguales en masa y \\
y fuego) y 3 principios (azufre, & a la idea de mezcla. & volumen. \\
mercurio y sal). & & \\
\hline
\end{tabular}

Cuadro 2 - Conceptualización de sustancia en los tres modelos históricos.

En este estudio histórico empezamos a comprender los problemas que tuvieron que superar los médicos paracelsianos como, por ejemplo, van Helmont en 1620 para mostrar la existencia de distintos "aires". O las de los filósofos mecánicos, como Boyle en 1661, al negar los elementos y principios aristotélico-escolásticos y llegar a clasificar los sistemas materiales en mezclas, "cuerpos perfectamente sin mezcla" (nuestras sustancias simples) y "cuerpos perfectamente mezclados" (los compuestos químicos actuales) (HOLTON; ROLLER, 1963). Es más, en el siglo XVIII todavía coexistían varios paradigmas filosóficos en la manera de ver el mundo entre los cuales estaban el modelo aristotélico-escolástico, todavía hegemónico, y varios modelos corpusculares de la materia. 
Los químicos, médicos, farmacéuticos, mineralogos y biólogos de este siglo se plantearon el problema práctico de la composición de los cuerpos mediante procesos de análisis y síntesis lo que facilitó la introducción del concepto macroscópico de sustancia como "cuerpo puro que tenía unas propiedades físicas y químicas características". Los historiadores Klein y Léfevre (2007) han puesto de relieve que el concepto de compuesto químico se presentó inicialmente en 1718 cuando Geoffroy publicó sus famosas "Tablas de afinidades electivas" que estableció empíricamente observando reacciones (de desplazamiento) entre ácidos y sales (BERTOMEU; GARCÍA-BELMAR, 2006). Aquellos historiadores concluyen que, posteriormente, en 1830, se asumió la idea ontológica de que el compuesto químico era una "sustancia estequiométrica". Es decir que tenía una composición química fija y definida por lo que se les denominaron "compuestos perfectos".

Podemos así vislumbrar las dificultades que pueden tener los estudiantes sobre la noción macroscópica de sustancia química derivadas de la ambigüedad con que se maneja en el lenguaje cotidiano el término sustancia: unas veces, se considera como sinónimo de "material o producto" - es decir, como mezcla -y otras veces como "una especie de sustancia simple de las que están hechas otras más complejas". En resumen, se puede afirmar que las enseñanzas de la Química no ha logrado incidir en los estudiantes sobre como clasificamos actualmente los materiales ordinarios, desde el punto de vista empírico, puesto que, al finalizar el Bachillerato científico, muchos de ellos siguen creyendo que la mezcla es lo mismo que la sustancia compuesta. Es más, en el siguiente apartado se mostrará como la enseñanza habitual al identificar sustancia simple y elemento químico contribuye a agudizar esta confusión entre mezcla y compuesto.

¿Para qué nos interesa a los profesores conocer la naturaleza de la ciencia y de la actividad cientifica?

La investigación didáctica ha puesto de relieve la importancia que tienen las concepciones inadecuadas del profesorado sobre la ciencia que se transmiten en la enseñanza. Un conocimiento actual de la HEC es también fundamental para tener una concepción más correcta de la naturaleza de la propia ciencia y para conocer las características de las estrategias de razonamiento que son propias de la actividad científica (McCOMAS, 1998). En otras palabras, al profesorado que enseñamos ciencia nos interesa, en particular, salir al paso de imágenes tópicas y erróneas sobre lo que es ciencia y sobre cómo se construye el conocimiento científico (FURIÓ, 1994). Hay ya muchos trabajos en los que se constata la existencia en el profesorado de visiones deformadas de la ciencia y del trabajo científico que se transmiten a través de la enseñanza (FERNÁN- 
DEZ et al., 2002; FURIÓ-MAS et al., 2011). A continuación se presentarán, a título de ejemplos, algunas de estas visiones deformadas transmitidas en la enseñanza que convendría tener en cuenta para evitar presentaciones didácticas inadecuadas en la enseñanza, en este caso, de Química.

\section{La visión acumulativa, de crecimiento lineal, de modelos históricos en la introducción del concepto de elemento químico}

Entre los profesores de Secundaria y Bachillerato es bien conocida la confusión que suele existir en los estudiantes acerca de las nociones de compuesto químico y mezcla como hemos visto en el apartado anterior. Algunos autores indican que el origen de este obstáculo se debe a la polisemia con que se utilizan en la vida cotidiana algunos términos como sustancia o elemento químico que pueden cambiar de significado según el contexto. Ahora bien, aunque una de las causas de las concepciones alternativas de los estudiantes puede ser el lenguaje cotidiano, queremos resaltar que otra explicación plausible puede consistir en que los profesores de Química introducimos estos conceptos desconociendo la naturaleza de estas construcciones científicas. Es decir, se desconoce el carácter evolutivo de los conceptos y no se suele saber que el significado de un concepto científico es dependiente del contexto de investigación (es decir, del modelo teórico en el que se idea). Se desconoce que en Química, al igual que en cualquier ciencia, se presentan modelos teóricos que tratan de interpretar la realidad y que cuando uno de estos modelos históricos cambia también suele cambiar el significado de los conceptos utilizados en dicho modelo. Así por ejemplo, en el paradigma aristotélico los cuatro elementos son ideados para explicar la gran diversidad de materiales existentes, cada uno con propiedades diferentes. En este contexto filosófico, cada uno de estos elementos abstractos era la representación mental de un par de propiedades generales que se le asignan a cada una de las 4 combinaciones binarias posibles (caliente-frío y seco-húmedo). En cambio, al pasar al contexto atomista del siglo XIX se idea una nueva ontología donde la noción de elemento químico para explicar la diversidad de materiales es bien distinto (cuadro 3). En este cuadro se presenta como se define el elemento químico en los modelos mecanicista (macroscópico), atomista (microscópico) y cuántico (submicroscópico).

En el caso de que el profesor posea una visión acumulativa acrítica, de crecimiento lineal, irá introduciendo las diferentes concepciones de elemento químico sin advertir a los estudiantes que cada definición se corresponde con un 


\begin{tabular}{|l|l|l|}
\hline \multicolumn{1}{|c|}{ Modelo mecanicista } & \multicolumn{1}{|c|}{ Modelo atomista } & \multicolumn{1}{c|}{ Modelo cuántico } \\
\hline Su programa de investigación & El elemento químico se & Se asocia la definición de \\
consistió en descubrir las sus- & define como el conjunto de & elemento químico al número \\
tancias simples. Estas sustan- & átomos iguales en masa y & atómico-carga nuclear-que \\
cias simples se asociaron (en & volumen. Con los elementos & no cambia en los procesos \\
nuestra opinión, indebida- & se explica la composición de & químicos. Idea que se deriva \\
mente) a elementos químicos & todas las sustancias (simples & del nuevo modelo cuántico \\
(las últimas sustancias del & o elementales y compuestas). & donde se explica la estructura \\
análisis químico). Asociación & electrónica de los átomos \\
asumida en la actualidad por la & & $\begin{array}{l}\text { y de los iones así como las } \\
\text { interacciones entre estas }\end{array}$ \\
I.U.P.A.C. & & especies químicas. \\
\hline
\end{tabular}

Cuadro 3 - Conceptualización de elemento químico en tres modelos históricos

modelo y al yuxtaponer estos modelos históricos de forma acrítica se presentarán dificultades (JUSTI; GILBERT, 2002). Una de las principales dificultades epistemológicas consistirá en identificar la sustancia simple y el elemento químico, como sucedió con los químicos seguidores de Lavoisier a finales del siglo XVIII y principios del XIX (ROCKE, 1986). El problema filosófico surgirá en la enseñanza cuando se superpongan los modelos mecanicista (macroscópico) y atomista (microscópico) puesto que el estudiante identificará la definición empírica de compuesto (sustancia única con propiedades características diferentes a las de las sustancias simples con las que se sintetizó) con la definición ontológica de "compuesto formado por dos elementos" del modelo atomista. Se inducirá así en los estudiantes el posible error de que el compuesto es una mezcla de dos sustancias simples. Esta superposición de significados se ha investigado y comprobado en una tesis doctoral reciente (LÓPEZ, 2008). Para salir al paso de esta dificultad se deberá poner en cuestión la identidad entre sustancia simple y elemento químico. Precisamente Mendeléyev en el Congreso de Karlsruhe (1860) se refirió a un segundo significado más "real" de elemento químico como especie de sustancia abstracta sin propiedades características (es decir, sin considerarla como sustancia simple) (SCERRI, 2008). Otra cosa es que con los átomos de un elemento químico se puedan formar las partículas de sustancias simples (elementales) y también de sustancias compuestas. Como consecuencia de esta identificación entre sustancia simple del modelo mecanicista (macroscópico) y elemento químico del modelo atomista (microscópico) que fue aceptada por la IUPAC en 1923 y que sigue actualmente asumida mayoritariamente por los químicos. Una prueba de que es así la tenemos al comprobar como en casi todos los libros de química se presenta la tabla periódica mezclando las propiedades características de una sustancia simple determinada (por ejemplo, el grafito) con las de un átomo del elemento (el C) que al unirse de cierta manera con otros iguales forman la estructura de dicha sustancia. 


\section{¿Se presenta una visión aproblemática y a-histórica al iniciar el estudio del equilibrio químico?}

Otro ejemplo de visión deformada de la ciencia que se transmite en la enseñanza es aquella que va directamente a exponer los conceptos sin tener en cuenta los problemas y modelos históricos que contribuyeron a su invención. Así por ejemplo, cuando en los libros de texto de Química se inicia el capítulo del Equilibrio químico se suele encontrar directamente un apartado titulado "El concepto de equilibrio químico" sin indicar previamente cuáles son los problemas cuya solución hizo necesaria la introducción de dicho concepto. Se olvida que los conceptos científicos son hipótesis que, en general, surgen del cuerpo teórico de conocimientos científicos con la finalidad de resolver algunos de los problemas que se plantearon históricamente. En el caso del equilibrio químico, se puede ir a la historia de la Química de finales del siglo XVIII. Fue en la última década de este siglo cuando se planteó la polémica entre Proust y Berthollet sobre qué era un compuesto. Esta polémica pasó inadvertida para la comunidad química de la época (BENSAUDE-VINCENT; STENGER, 1998) y se dirimió históricamente aceptando tanto los compuestos perfectos de Proust como los imperfectos de Berthollet. Pero en el fondo de aquella controversia también se planteaba cuál era el significado de reacción química. Para Berthollet, todas las reacciones eran incompletas y presentaba como ejemplo prototípico de reacción incompleta la disolución acuosa del nitro de Chile que trataba de optimizar en su trabajo. El estudio de estas reacciones incompletas abrió una nueva línea de investigación que obtuvo resultados importantes 70 años después cuando se publicó el artículo "Sobre las afinidades" de Guldberg y Waage en 1864, cuyo contenido se conoce más como la ley de acción de masas o del equilibrio químico.

\section{Breve presentación de dos tradiciones de investigación en el aprendizaje de las Ciencias}

Ya hemos visto en el apartado anterior como la historia y filosofía de la ciencia es fundamental para el profesor ya que le puede servir para seleccionar los contenidos de la disciplina, predecir las principales dificultades del alumnado y, en particular, salir al paso de visiones deformadas de la ciencia. También lo es para conocer las características de las formas de razonar en la actividad 
científica con el fin de seleccionar estrategias didácticas a desarrollar en la educación científica (McCOMAS, 1998). Una de las principales características de la investigación científica es precisamente la de resolver problemas. Si la educación quiere lograr una adecuada alfabetización científica y tecnológica, no puede renunciar a facilitar al estudiante las competencias básicas (conocimientos, habilidades y valores) necesarias para resolver problemas en la vida real. Es, pues, importante conocer la investigación sobre los distintos modelos de aprendizaje existentes para poder seleccionar uno y decidir coherentemente el método de enseñanza que vamos a utilizar en el aula. Por ello presentaremos, en primer lugar, una descripción breve de dos tradiciones de investigación en el aprendizaje y, en segundo lugar, las orientaciones metodológicas del modelo de aprendizaje que venimos experimentando desde hace un par de décadas en las clases de Física y Química en la Educación Secundaria (12 a 16 años) y en el Bachillerato (16 a 18 años).

¿Cuáles son las principales tendencias de investigación en el aprendizaje de las ciencias?

En un reciente manual de investigación, Handbook of Research on Science Education (2007), encontramos sendos capítulos que presentan revisiones sobre las tradiciones de investigación en el aprendizaje de las ciencias. En el primero de estos capítulos, Anderson (2007) indica que la investigación sobre aprendizaje en ciencias puede ser ampliamente caracterizada por estar centrada en el desarrollo de la alfabetización científica concebida como conjunto de conocimientos, destrezas y valores que esperamos adquieran los estudiantes cuando aprenden ciencias (sin conseguirlo). A continuación, el autor presenta varias tradiciones en este tipo de investigaciones en didáctica de las ciencias tales como la del cambio conceptual, la sociocultural y la critica.

Tradiciones que el autor caracteriza y compara seleccionando aspectos comunes del aprendizaje de la ciencia que suelen estar implícitos o explícitos en los estudios sobre esta temática tales como: las ideas sobre los alumnos y el aprendizaje de las ciencias, ideas sobre la naturaleza de la ciencia (concebida como subcultura que comprende lenguaje, valores y prácticas especializadas), metas y métodos de investigación e ideas para mejorar el aprendizaje.

La alfabetización científica basada en el cambio conceptual es la que tiene el modelo más potente que usa las ideas y métodos desarrollados por Piaget. Este modelo se caracteriza por considerar: la ciencia como un diálogo teorético con la naturaleza; el alumno como un pensador racional pero inexperto (con sus "ecologías conceptuales" en tópicos específicos); la investigación está centrada en las concepciones de los estudiantes y los métodos de enseñanza tienen como 
finalidad conseguir el cambio conceptual a través de situaciones de conflicto cognitivo entre los esquemas desarrollados por la experiencia de los estudiantes y los esquemas conceptuales científicos.

La tradición de investigación sociocultural es aquella que considera la alfabetización científica como participación en una comunidad centrada en el discurso. Se basa más en las ideas de Vygotsky y sus seguidores y se caracteriza por: considerar a la ciencia como una comunidad de discurso; el alumno aprende gracias a su participación en actividades en las que se plantean discursos diferentes; por ello, las investigadores se centran en el análisis de la cultura, el lenguaje y las prácticas de los estudiantes y de las comunidades científicas. Los métodos de enseñanza prestan atención a la comunicación y negociación entre el discurso cotidiano y el científico para favorecer la superación del conflicto sociocultural.

Finalmente, la perspectiva crítica concibe la alfabetización como elemento en contra de la posible alienación del estudiante por la cultura y la ideología dominantes en la escuela. En esta tradición la ciencia se considera una institución ideologizada, el aprendizaje de la ciencia se presenta como adoctrinamiento en el desarrollo de la conciencia crítica y los métodos de enseñanza tienen como finalidad conseguir una alfabetización crítica.

Remitimos al lector a dicho manual para ver las ventajas y limitaciones de cada una de aquellas tradiciones.

Por otra parte, en un segundo capítulo del manual citado en el inicio de este subapartado, Scott et al. (2007) presentan una revisión de las concepciones de los alumnos y el aprendizaje conceptual en ciencias que partiendo del concepto general de aprender justifican las tradiciones del cambio conceptual y la sociocultural para acabar mostrando, entre otros, el actual enfoque socioconstructivista del proceso de enseñanza-aprendizaje de las ciencias que propugnan (LEACH; SCOTT, 2002). Ahora bien, resulta muy interesante leer como los autores, a la hora de estructurar su revisión sobre los diferentes enfoques de aprendizaje de las ciencias, toman como criterio dos metáforas clave para conceptualizar el aprender usadas por Sfard (1998): la metáfora adquisición y la metáfora participación. Esta autora indica que en cualquier época el aprendizaje humano ha sido considerado como posesión o adquisición de algo - por ejemplo, un concepto - que queda almacenado en la cabeza del aprendiz. Este enfoque del aprendizaje de conceptos como proceso de adquisición cognitiva pone el énfasis en su carácter individual que está implícito en cualquier modelo pero, especialmente, está explícito en el de la tradición del cambio conceptual que comenzó en la década de los 80 del siglo pasado. Por el contrario, la metáfora participación ofrece una nueva perspectiva de aprender donde el aprendiz ha de ser visto como una persona interesada en participar en ciertas actividades 
más que en acumular posesiones privadas. Es decir, esta perspectiva predomina en todos aquellos enfoques de aprendizaje en los que se enfatiza alguna forma de participación. O sea, como indica la misma autora: "learning a subject is now conceived of a becoming a member of a certain community" (p. 6). En este sentido, los enfoques de enseñanza-aprendizaje de las ciencias basados en la indagación ("inquiry-based approaches") se justifican teóricamente dentro de esta segunda perspectiva como contextos educativos en los que los alumnos forman parte de una comunidad de práctica científica cuya finalidad es adquirir, entre otras destrezas, las relativas a la investigación científica (saber plantear problemas, emitir hipótesis para su solución y ponerlas en cuestión mediante experimentación) y el aprendizaje del lenguaje social de la ciencia escolar (MORTIMER; SMOLKA, 2003). Ahora bien, estos dos tipos de perspectivas de enfoques del aprendizaje no hay que concebirlos como extremos opuestos de un continuo sino que cualquier enfoque tendrá en mayor o menor medida las dos metáforas. Por ejemplo, en una visión socioconstructivista del aprendizaje de conceptos se parte de la perspectiva del aprendizaje como adquisición cognitiva pero también se admite que el contexto social es parte integral del proceso de aprendizaje. Una vez decidido el modelo de aprendizaje que creemos más eficaz, será importante decidir las orientaciones y estrategias metodológicas a desarrollar en el aula.

\section{Aspectos metodológicos que se tienen en cuenta en el modelo de aprendizaje como investigación orientada}

Se trata de plantear una propuesta de aprendizaje como investigación orientada (GIL et al., 1991; FURIÓ, 2001; GUISASOLA et al., 2008) que, como sugiere el informe Rocard et al. (2007), debería ser la nueva pedagogía para el futuro de la enseñanza de las ciencias en Europa. Este modelo que venimos desarrollando tiene como objetivo aproximar el aprendizaje de los estudiantes a las estrategias del trabajo científico y en ellas se han de poder integrar de forma natural las dimensiones social, histórica, filosófica, etc. de la vida actual.

En esta propuesta los estudiantes realizan pequeñas investigaciones para resolver problemas de interés. Cuando un alumno se plantea un problema que le interesa y trata de resolverlo científicamente, en general, ha de precisarlo, emitir unas hipótesis, elaborar unas estrategias de resolución, etc., y analizar cuidadosamente los resultados y sus posibles consecuencias. Es precisamente en este proceso, adecuadamente impulsado y orientado por el profesor, cuando 
pueden aparecer de manera funcional (si es que existen) las posibles concepciones alternativas y la consiguiente necesidad de modificarlas haciendo posible la evolución de tales ideas hacia las ideas científicas que se quieren enseñar.

Para esta propuesta, es conveniente la estructuración de la clase en pequeños grupos, porque favorece el nivel de participación y la creatividad necesaria para la emisión de hipótesis, realización de diseños, etc. que se plantean en las actividades, seguidas de puestas en común (que no debe emplear excesivo tiempo) y de intervenciones del profesor, para realizar reformulaciones globalizadoras de las aportaciones de los grupos o incluso - aún cuando éstas sean incompletas - para añadir información. Esto no supone una trasgresión del método propuesto. El hecho de que los estudiantes hayan abordado previamente las actividades hace que su receptividad ante la información sea superior, por responder a cuestiones que ellos se han planteado. El propósito de los programas de actividades es evitar la tendencia espontánea a primar la actividad del profesor, es decir, a centrar el trabajo de clase en el discurso ordenado del profeso. Todo ello no excluye las diferentes mediaciones que ha de realizar el profesor ni tampoco que alguna actividad pueda consistir en escuchar una exposición del mismo profesor o en la lectura de un texto (para extraer las ideas clave, comentar, etc.)

El programa de actividades elaborado por el profesor (o, mejor aún, por el grupo de profesores que imparten la disciplina en el mismo Centro) ha de incluir estrategias de aprendizaje semejantes a las de la actividad científica que no son tenidas en cuenta en la enseñanza habitual de las ciencias, como:

- La discusión del posible interés de las situaciones problemáticas propuestas que dé sentido a su estudio, incluyendo la necesaria toma de decisiones acerca de la conveniencia de abordar dicho estudio en el aula;

- El estudio cualitativo de las situaciones problemáticas abordadas, que ayude a comprenderlas y a acotarlas a la luz de los conocimientos disponibles;

- La invención de conceptos y emisión de hipótesis fundamentadas, que permitan orientar el tratamiento científico de los problemas;

- La elaboración y puesta en práctica de estrategias de resolución, incluyendo el diseño y realización de montajes experimentales para poner en cuestión las hipótesis emitidas;

- El análisis y comunicación de los resultados obtenidos, cotejándolos con los de otros grupos de estudiantes y con los de la comunidad científica; 
- La consideración de las posibles perspectivas de los conocimientos construidos: planteamientos de nuevos problemas, conexión con otros conocimientos, elaboración de productos, implicaciones sociales y ambientales, etc.

Es preciso resaltar que esta familiarización con la metodología científica, no puede resolverse de manera parcelada o limitada a los trabajos prácticos. Para que el cambio metodológico pueda llevarse a cabo, es necesario que se extienda a todas las actividades claves en la enseñanza de las ciencias, desde la introducción de conceptos a los trabajos prácticos pasando por la misma resolución de problemas de papel y lápiz. Además, señalar que este modelo también integra la dimensión axiológica del aprendizaje al tener en cuenta las relaciones CTSA (SOLBES; VILCHES, 1997) en el interés de la situación problemática abordada, en las posibles perspectivas, etc.

Finalmente, los nuevos conocimientos adquiridos en el escenario antes esbozado han de mostrar su utilidad en una variedad de situaciones, resaltando, en particular, las relaciones Ciencia, Tecnología, Sociedad y Ambiente y valorando las posibles consecuencias del desarrollo tecnocientífico así como la necesaria toma de decisiones personales ante los problemas de sostenibilidad que se puedan presentar.

Estas características son compartidas con otros modelos de enseñanza-aprendizaje como investigación en la escuela (NACIONAL RESEARCH COUNCIL, 2000). Modelos que se van imponiendo hasta en la propia enseñanza universitaria como se puede apreciar en artículos publicados en Nature (POWELL, 2003).

\section{Consideraciones finales}

En síntesis, la cuestión básica de para qué le puede interesar a un profesor investigador o innovador conocer la historia y epistemología de la Química se ha contestado resaltando que para poder fundamentar, en síntesis, el diseño de secuencias de enseñanza o del curriculum de Química. Por varias razones, entre las cuales destacamos las siguientes: La realización del análisis histórico y epistemológico del conocimiento científico es fundamental para la primera fase de elaboración de una unidad didáctica, esto es para idear un hilo conductor con el que organizar y secuenciar los contenidos de una secuencia de enseñanza (por ejemplo, los de una unidad didáctica) (LEACH; SCOTT, 2002). También para 
tomar decisiones respecto a qué estrategias didácticas utilizar en el proceso de enseñanza que se va a seguir (en particular, la metodología de enseñanza a utilizar en el aula) así como las dificultades de aprendizaje que puedan surgir. Para decidir estas estrategias didácticas será importante que el profesorado conozca los modelos de aprendizaje de las principales tradiciones de alfabetización científica que se han descrito por varios investigadores en didáctica de las ciencias.

En este último sentido, queremos manifestar que, como indican filósofos e historiadores, necesitamos conocer nuestro pasado en didáctica de las ciencias para poder comprender el presente, para comprender a nuestros estudiantes y para conocernos a nosotros mismos. Esta comprensión implica no solamente saber cómo es el mundo sino también saber cómo construimos conocimiento y cómo razonamos científicamente. Estos conocimientos y habilidades son necesarios no sólo para situarnos en este mundo sino también para tomar decisiones con el que afrontar colectivamente los problemas del futuro. De un futuro que ha de ser, por necesidad, cada vez más sostenible, más justo y más solidario.

\section{REFERENCIAS}

ABELL, S. K.; LEDERMAN, N. G. Handbook of Research on Science Education. New York: Routledge, 2007.

ANDERSON, C. Perspectives on Science Learning. En: ABBEL, S. K.; LEDERMANN. G. Handbook of Research on Science Education. New York: Routledge, 2007, p. 3-30.

BENSAUDE VINCENT, B.; STENGERS, I. Historia de la Química. Madrid: Addison-Wesley, Universidad Autónoma de Madrid, 1998.

BERTOMEU, J. R.; GARCIA-BELMAR, A. La revolución química. Entre la historia y la memoria. Valencia: Publicaciones de la Universitat de València, 2006.

BYBEE, R. Towards an Understanding of Scientific Literacy. En: GRAEBER, W.; BOLTE, C. (Eds.). Scientific Literacy. Kiel: IPN, 1997.

DOMÍNGUEZ SALES, M. C.; FURIÓ-MÁS, C. Aprendiendo de la historia y filosofía de la ciencia: Deficiencias en la enseñanza de los conceptos macroscópicos de sustancia y compuesto químico. Actas del VII Congreso Internacional sobre Investigación en Didáctica de las Ciencias. Comunicación oral. Septiembre. Granada, 2001.

ERDURAN, S.; DUSCHL, R. Interdisciplinary characterizations of models and the nature of chemical knowledge in the classroom. Studies in Science Education, v. 40, p. 105-138, 2004. 
FERNÁNDEZ, I.; GIL, D.; CARRASCOSA, J. Visiones deformadas de la ciencia transmitidas por la enseñanza: una revisión bibliográfica. Enseñanza de las Ciencias, v. 20, n. 3, p. 477-488, 2002.

FILLON, P. Histoire des sciences et refléxion épistémologique des élèves. Aster, v. 12, p. 91-120, 1991.

FURIÓ, C. Tendencias actuales en la formación del profesorado de Ciencias. Enseñanza de las Ciencias, v. 12, n. 2, p. 188-199, 1994.

. La enseñanza-aprendizaje de las ciencias como investigación: un modelo emergente. En: GUISASOLA, J.; PÉREZ DE EULATE, L. (Eds.). Investigaciones en didáctica de las ciencias experimentales basadas en el modelo de enseñanza-aprendizaje como investigación orientada. Bilbao: Servicio Editorial. Universidad del País Vasco, 2001.

FURIÓ-MÁS, C. Contribuciones de la historia y filosofía de la ciencia a la enseñanza-aprendizaje de la química. Journal of Science Education, v. 10, p. 34-36, 2009.

FURIÓ, C.; AZCONA, R.; GUISASOLA, J. La enseñanza de los conceptos de "cantidad de sustancia" y de "mol" basada en un modelo de aprendizaje como investigación orientada. Enseñanza de las Ciencias, v. 24, n. 1, p. 43-58, 2006.

; BULLEJOS, J.; DE MANUEL, E. L'apprentissage de la réaction chimique comme activité de recherche. Aster, v. 18, p. 141-168, 1994.

; DOMÍNGUEZ, M. C. Problemas históricos y dificultades de los estudiantes en la conceptualización de sustancia y compuesto químico. Enseñanza de las Ciencias, v. 25, n. 2, p. 241-258, 2007.

FURIÓ-MÁS, C.; FURIÓ-GOMÉZ, C. ¿Cómo diseñar una secuencia de enseñanza de ciencias con una orientación socioconstructivista? Educación Química, p. 246-251, junio 2009.

; SOLBES-MATARREDONA, J. How are the concepts of internal energy and enthalpy presented in Chemistry textbooks? Distorted views of science and scientific activity in Thermochemistry. Proceedings of 2011 ESERA Conference. September, 5-9. Lyon (France), 2011.

FURIÓ, C.; HERNÁNDEZ, J.; HARRIS H. Parallels between adolescents' conception of gases and the History of Chemistry. Journal of Chemical Education, v. 64, n. 7, p. 616-618, 1987.

; DOMINGUEZ, C.; AZCONA, R.; GUISASOLA, J. La enseñanza y el aprendizaje del conocimiento químico. En PERALES, F. J.; CAÑAL, P. (Dir.); Didáctica de las Ciencias Experimentales. Teoría y Práctica de la Enseñanza de las Ciencias. Alcoy: Editorial Marfil, 2000, p. 421-450.

GIL, D.; CARRASCOSA, J.; FURIÓ, C.; MARTÍNEZ-TORREGOSA, J. La Enseñanza de las Ciencias en la Educación Secundaria. Barcelona: Horsori-ICE Universidad de Barcelona, 1991. 
FURIÓ-MÁS, C.; FURIÓ-GÓMEZ, C.; SOLBES-MATARREDONA, J. Profundizando en la...

GUISASOLA, J.; FURIÓ, C.; CEBERIO, M. Science Education Based on Developing Guided Research. En: THOMASE, M. V. (Ed.). Science Education in Focus. New York: Nova Science Publishers, Inc., 2008, p. 173-202.

HOLTON, G.; ROLLER, R. Introducción a la Física Moderna. Barcelona: Ed. Reverté, 1963.

JENSEN, W. B. Logic, History, and the Chemistry Textbook. I. Does Chemistry Have a logical Structure? Journal of Chemical Education, v. 75, n. 6, p. 679-687, 1998.

JUSTI, R.; GILBERT. J. K. Models and modelling in chemical education. En: GILBERT, J.K. et al. (Eds.). Chemical Education: Towards Research-base Practice. Dordrecht: Kluwer Academic Publishers, 2002, p. 47-68.

KLEIN, U.; LÉFEVRE, W. Materials in eighteenth-century science: a historical ontology. Cambridge, Mass.: MIT Press, 2007.

KUHN, T. S. La estructura de las revoluciones cientificas. México: Fondo de Cultura Económica, 1971.

LAUGKSCH, R. C. Scientific literacy. Science Education, v. 84, n. 1, p. 71-94, 2000.

LEACH, J.; SCOTT, P. Designing and evaluating science teaching sequences: an approach drawing upon the concept of learning demand and a social constructivist perspective on learning. Studies in Science Education, v. 38, p. 115-142, 2002.

LÓPEZ, D. M. La enseñanza y el aprendizaje del concepto de elemento químico. Análisis crítico y propuesta de mejora. Tesis doctoral (Departament de Didàctica de les Ciències Experimentals i Socials) - Universitat de València, 2008.

McCOMAS, W. F. (Ed.). The nature of science in science education. Rationales and strategies. Dordrecht: Kluwer Academic Publishers, 1998.

MILLAR, R.; OSBORNE, J. Beyond 2000: Science Education for the Future. London: KCL, 1998.

MORTIMER, E. F.; SMOLKA, A. L. (Eds.). In: ENCONTRO INTERNACIONAL LINGUAGEM, CULTURA E COGNIÇÃO, 2., Campinas, 2003. Anais... Campinas: Faculdade de Educação de Unicamp, 2003.

NATIONAL RESEARCH COUNCIL. National Science Education Standards. Washington, D.C.: National Academy Press, 1996.

. Inquiry and the National Science Education Standards. A Guide for Teaching and Learning. Washington D.C.: National Academia Press, 2000.

OSUNA, L.; MARTÍNEZ-TORREGROSA, J.; CARRASCOSA, J.; VERDÚ, R. Planificando la enseñanza problematizada: El ejemplo de la óptica geométrica en la educación secundaria. Enseñanza de las Ciencias, v. 25, n. 2, p. 277-294, 2007. 
POSNER, G. J.; STRIKE, K. A.; HEWSON, P. W.; GERTZOG, W. A. Accomodation of a scientific conception: toward a theory of conceptual change. Science Education, v. 66 , p. 211-227, 1982.

POWELL, K. Spare me the lecture. Nature, v. 425, p. 234-237, 2003.

ROCARD, M. et al. Science education Now: A renewed Pedagogy for the future of Europe. European Communities: Belgium, 2007. Disponible en: < http://ec.europa.eu/ research/science-society/document_library/pdf_06/report-rocard-on-science-education en.pdf $>$. Acceso en: 15/10/2011.

ROCKE, A. J. Chemical Atomism in the Nineteenth Century. From Dalton to Cannizzaro. Columbus: Ohio State University Press, 1986.

SCERRI, E.R. La Tabla Periódica. Investigación y Ciencia, p. 50-56, abril 2008.

SCOTT, P.; ASOKO, H.; LEACH, J. Student Conceptions and Conceptual Learning in Science. En: ABELL, S. K.; LEDERMAN, N. G. Handbook of Research on Science Education. New York: Routledge, 2007, p. 31-56.

SFARD, A. On two metaphors for learning and the dangers of choosing just one. Educational Researcher, v. 27, n. 2, p. 4-13, 1998.

SOLBES, J.; MONTSERRAT, R., FURIÓ, C. El desinterés del alumnado hacia el aprendizaje de la ciencia: implicaciones en su enseñanza. Didáctica de las Ciencias Experimentales y Sociales, v. 21, p. 91-117, 2007.

; VILCHES, A. STS interactions and the teaching of physics and chemistry. Science Education, v. 81, n. 4, p. 377-386, 1997.

WANDERSEE, J. H.; MINTZES, J. J.; NOVAK, J. D. Research on alternative conceptions in science. In: GABEL, D.J. (Ed.). Handbook of Research on Science Teaching and Learning, New York: MacMillan Publishing Co., 1994, p. 188.

Texto recebido em 23 de novembro de 2011.

Texto aprovado em 14 de dezembro de 2011. 